\title{
Lack of a Dose Response from 7 Days of Ischemic Preconditioning in Moderately trained Cyclists
}

()(1) $\odot$

\author{
Authors \\ Angus Lindsay ${ }^{1}$, Carl Petersen ${ }^{2}$, Hamish Ferguson ${ }^{3}$, Gavin Blackwell ${ }^{4}$, Stephen Rickerby ${ }^{5}$
}

\section{Affiliations}

1 Program in Physical Therapy and Rehabilitation Sciences, University of Minnesota Twin Cities, Minneapolis, United States

2 School of Health Sciences, University of Canterbury, Christchurch, New Zealand

3 Sport Performance Research Institute, Auckland University of Technology, Auckland, New Zealand

4 School of Sciences and Physical Education, University of Canterbury, Christchurch, New Zealand

5 UC Sport, University of Canterbury, Christchurch, New Zealand

\section{Key word}

time trial, hypoxia, occlusion, reperfusion, sport

$\begin{array}{ll}\text { received } & 06.02 .2018 \\ \text { revised } & 23.05 .2018 \\ \text { accepted } & 25.05 .2018\end{array}$

\section{Bibliography}

DOI https://doi.org/10.1055/a-0639-5035

Sports Medicine International Open 2018; 2: E91-E97

(c) Georg Thieme Verlag KG Stuttgart · New York

ISSN 2367-1890

\author{
Correspondence \\ Dr. Carl Petersen, \\ School of Health Sciences \\ University of Canterbury \\ Private Bag 4800 \\ 8140 Christchurch \\ New Zealand \\ Tel.: + 64/336/93438 \\ carl.petersen@canterbury.ac.nz
}

\begin{abstract}
Ischemic preconditioning (IP) has a small benefit on exercise performance, but differences in the IP method, performance tasks and exercise modality have made providing practical coach guidelines difficult. We investigated the performanceenhancing effects of IP on cyclists by comparing the frequency of IP application over a 7-day period. Using a randomized, sham-controlled, single-blinded experiment, 24 competitive age-group track cyclists ( $38 \pm 12$ years) were assigned to one of three twice-daily (sham: 20 and $20 \mathrm{mmHg}$; once-a-day: 20 and $220 \mathrm{mmHg}$; twice-a-day: 220 and $220 \mathrm{mmHg}$ ) IP leg protocols ( $4 \times 5 \mathrm{~min}$ ischemia/ $5 \mathrm{~min}$ reperfusion alternating between legs) over seven consecutive days. A 4000-m cyclingergometer time trial was completed before, immediately following and one week after the protocols. Neither mean power, nor 4000-m performance time nor $\mathrm{VO}_{2}$ were significantly affected by either of the IP protocols compared to the sham at any time point following treatment. Repeated application of IP over seven days did not enhance the performance of trained cyclists in a 4000-m laboratory time trial. More research is required to understand how changes to methodological variables can improve the chances of IP successfully enhancing athlete performance.
\end{abstract}

Ischemic preconditioning (IP) is gaining popularity as a pre-exercise intervention to provide acute meaningful improvements in competitive sporting performance. Although the effect of IP is still debated, a typical exercise IP protocol subjects the local musculature to $4 \times 5 \mathrm{~min}$ ischemia followed by $5 \mathrm{~min}$ of reperfusion [26]. A single exposure to IP has shown positive benefits on aerobic and anaerobic exercise [28], but unclear effects with regard to improving sprint and power-based performance [28] and no improvement over placebo in resistance exercise repetition performance [21, 22] or recreational sprint swimming [20]. A recent systematic review by Incognito and colleagues (2016) concludes that "although, large between-study variability exists, the most consistent benefit of IP 
is for an improvement in time trial performance in exercise tests of predominantly lactic anaerobic and aerobic capacity" [14]. Similarly, a meta-analysis by Salvador et al. (2016) calculates IP to have a small beneficial effect $(\mathrm{ES}=0.43 ; 90 \% \mathrm{Cl}, 0.28-0.51$ ) on performance and that IP had a $>99 \%$ and $~ 58 \%$ chance of benefiting aerobic and anaerobic exercise, respectively [28]. In terms of the magnitude of improvement, during 5-km cycling time-trial performances ParadisDeschenes et al. (2017) noted a $1.1 \%$ and $1.5 \%$ improvement at low and moderate altitudes, respectively [25].

Despite the popularity of a singular application of IP, the effect of daily repeated ischemia-reperfusion has only recently been investigated. Using a randomized cross-over trial, Foster et al. (2014) investigated the prophylactic use of IP administered daily over 5 days and found improved oxygen saturations following ascent to high altitude [10]. Similarly, 7 days of IP application improved flowmediated dilation and resting skin microcirculation and these beneficial effects remain present for at least 7 days after the cessation of the IP stimulus [16].

In an exercise context, one recent study by Banks et al. (2016) with 9 days of IP failed to show any improvement in aerobic capacity during a progressive ramp bicycle test conducted at least $24 \mathrm{~h}$ after the final IP application [2]. However, another study using 7 days of IP resulted in significant improvements in maximal oxygen consumption $\left(\mathrm{VO}_{2}\right.$ max $)$ and maximum aerobic power in untrained individuals [19], and it was also shown that aerobic capacity continued to increase seven days following the cessation of treatment, which is similar to that shown in endothelial function and microcirculation [16]. Moreover, the amount of ischemia-reperfusion tissue exposure area and time required to elicit these changes were similar to the majority of exercise-related research $(4 \times 5 \mathrm{~min})$.

\section{Repeating the IP}

Coaches and athletes are interested in the optimal IP dose to maximize exercise capacity and sport-specific performance. Recently a dose comparison study confirmed the traditional $(4 \times 5 \mathrm{~min})$ protocol was superior for improving endurance performance when compared to a doubling of the ischemia-reperfusion-induced cellular stress $(8 \times 5 \mathrm{~min})$ within the same session [6]. This leads to the question of whether increasing the cellular stress through a combination of daily application and an increased daily frequency of application would prove more successful.

Within medicine there is emerging evidence for a dose dependency of daily IP application on clinical outcomes [16]. This research intends to explore dose dependency in the sporting context. We measured several aerobic capacity parameters during a simulated 4000-m cycling time-trial time with the intention of elucidating any potential added benefit of doubling the ischemia-reperfusion tissue exposure in moderately trained cyclists. Our hypothesis was that seven days of a twice-a-day alternating-leg unilateral repeated IP protocol would provide enhanced aerobic capacity and sportspecific benefit over a once-a-day repeated IP protocol.

\section{Materials and Methods}

Subjects Twenty-six age-group track cyclists competing at the national level in their respective categories volunteered for the study.
Two withdrew for different reasons resulting in 24 ( 13 men and 11 women) being included in the full analysis. The inclusion criteria were track cycling athletes who were currently competing nationally in their respective age group. Athletes who had previously undergone surgery to alleviate iliac artery endofibrosis were excluded from the study as a precaution to the reperfusion-based protocol and pressure cuff placement, as well as any athlete who was currently taking or had taken creatine or beta-alanine supplements in the previous four weeks. We confirm that our study meets the ethical standards of the Sports Medicine International Open journal [12]. The experimental protocol was approved by the University of Canterbury Human Ethics Committee (approval number HEC 2015/78) and all athletes were informed of the risks involved in the study before their written consent was obtained.

\section{Study design}

We conducted a randomized, sham-controlled, single (athlete) blinded experiment to evaluate the effects of repeated IP dose-dependence on cycling aerobic capacity and performance ( $\mathbf{F i g . 1}$ ). All athletes were competing at the national age-group level and were accustomed to the specific testing protocols of the study, meaning no familiarization of the testing procedures was necessary. Upon reporting to the lab, athletes had their height measured with a wall-mounted stadiometer (Seca, Hamburg, Germany) and body mass recorded (inBody270, Seoul, Korea) before undertaking a 4000-m cycling time trial. Following the completion of this first 4000-m time trial, athletes were randomly assigned to either once-a-day or twice-a-day or sham IP protocols. Randomization was achieved by ranking and matching athletes based on their $\mathrm{VO}_{2}$ peak and using a coin toss to allocate into groups with their characteristics provided in $>$ Table 1 . Although athletes were likely aware of pressure differences between conditions, they remained naïve to the rationale of the experiment. At the completion of the first testing day, each athlete was required to complete one of three repeated IP protocols for the next seven consecutive days before returning the following Sunday to complete the same testing procedure. This was followed by seven days of no IP treatment before repeating the test for a final time.

\section{Testing procedures}

\section{0-m cycling-ergometer time trials}

The experimental protocol required athletes to complete a 4000$\mathrm{m}$ cycling-ergometer time trial with re-testing completed a week and a fortnight later. For logistical reasons the athletes were tested in the same order, each individual was tested at approximately the same time of day, and all athletes performed the time trial alone.

Athletes were instructed to refrain from any form of strenuous or prolonged exercise, caffeine, supplements (sodium bicarbonate) and alcohol for at least $24 \mathrm{~h}$ prior to testing. They were asked to remain in a euhydrated state and to adhere to their normal diet and training regimes or activities throughout the two weeks of testing. There was no effort to control diet or training, although each athlete was asked to provide a training diary for the study duration. Data collection was undertaken at the beginning of the track season; hence the subjects were all completing a strength/aerobic phase as indicated in $>$ Table 1. 


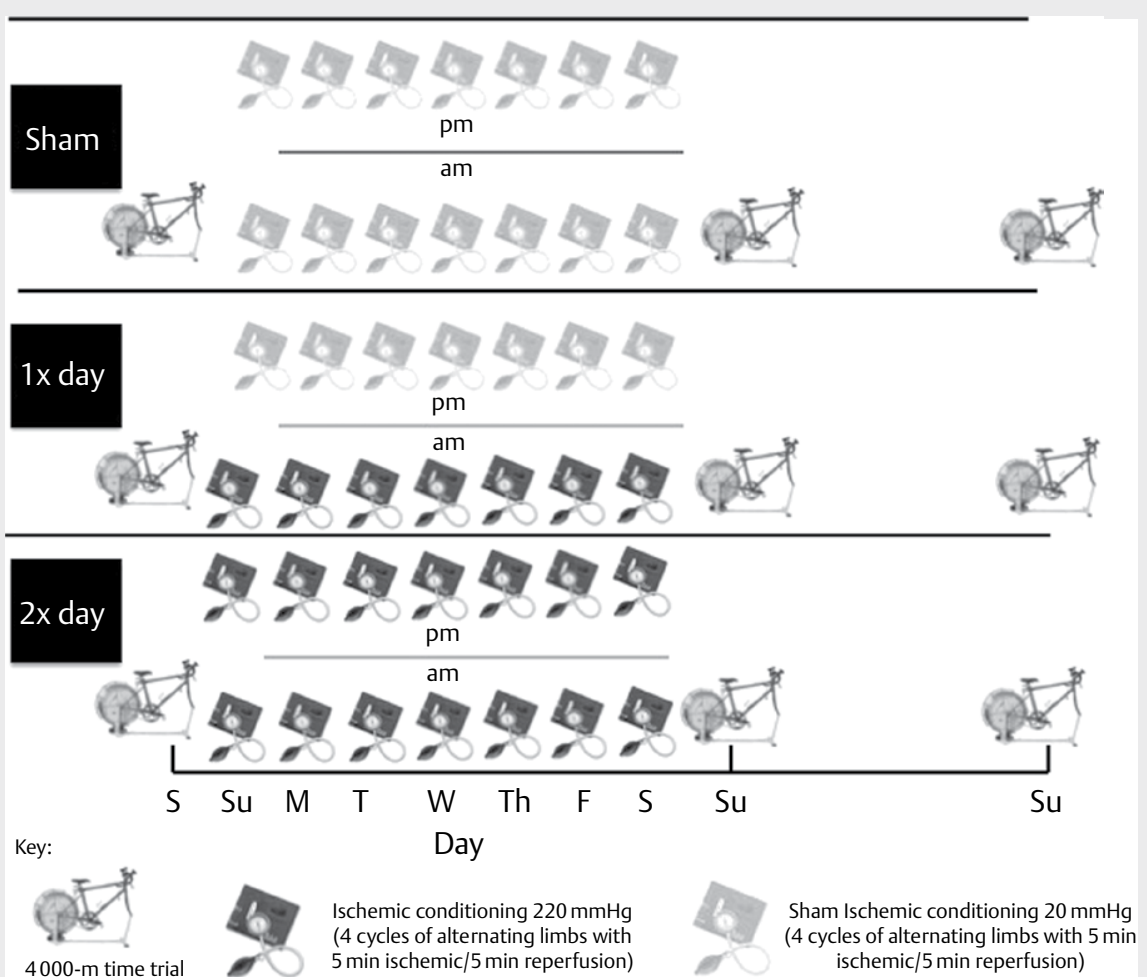

- Fig. 1 Experimental design

- Table 1 Subject characteristics. Data is presented as mean \pm SD.

\begin{tabular}{|l|l|l|l|}
\hline & Sham $(\mathbf{n}=\mathbf{8})$ & Once-a-day $(\mathbf{n}=\mathbf{8})$ & Twice-a-day $(\mathbf{n}=\mathbf{8})$ \\
\hline Age $($ years $)$ & $41.0 \pm 10.1$ & $36.0 \pm 17.9$ & $36.0 \pm 10.3$ \\
\hline Height $(\mathrm{cm})$ & $170.4 \pm 10.4$ & $173.6 \pm 6.7$ & $177.6 \pm 7.9$ \\
\hline Weight $(\mathrm{kg})$ & $70.6 \pm 6.9$ & $72.7 \pm 13.7$ & $82.2 \pm 13.8$ \\
\hline $\mathrm{VO}_{2}$ peak $\left(\mathrm{ml}^{\mathrm{m}} \mathrm{min}^{-1} \cdot \mathrm{kg}^{-1}\right)$ & $45.8 \pm 13.9$ & $49.5 \pm 8.8$ & $51.1 \pm 10.9$ \\
\hline Training hours per week & $13.7 \pm 7.1$ & $11.3 \pm 3.9$ & $10.4 \pm 8.9$ \\
\hline Training phase & Strength/aerobic & Strength/aerobic & Strength/aerobic \\
\hline
\end{tabular}

The 4000-m cycling time trial is considered the pinnacle of aerobic track-cycling performance. It consists of a self-pacing strategy that predominantly utilizes aerobic energy systems except for a greater (10\%) anaerobic contribution at the beginning and end of the effort [31]. Most importantly, it offers a reliable platform for assessing aerobic capacity considering trained cyclists use a highly consistent pacing strategy [31]. Athletes were required to complete their traditional warm-up strategy on a Monark ergometer (Ergomedic 894E, Monark, Vansbro, Sweden) that replicated their competition preparation on each of the three occasions. The 4000 $\mathrm{m}$ time trial was completed on a Velotron Dynafit cycle ergometer (Racermate, Seattle, WA, USA) which has been shown to produce consistent indices of power during exercise [30]. Before each test commenced, factory calibration was verified using the Accuwatt “run down" verification program (RacerMate, Seattle, WA, USA).
Each athlete had the ergometer specifically adjusted to replicate their own bike, which included their own pedals. The settings, which included crank length, seat height, top tube length, handle bar height and bottom bracket distance, were recorded and replicated on each occasion. At the completion of the warm-up, athletes were given 5 mins to relax before completing the flat 4000-m time-trial profile using the provided software (Racermate, Seattle, WA, USA). No visual feedback or verbal encouragement was provided except for verbal feedback every $250 \mathrm{~m}$ to replicate an indoor 250-m velodrome setting. Athletes were instructed to complete the $4000 \mathrm{~m}$ in the quickest time possible using a pre-selected gear (same on each occasion) that they would typically utilize in competition. The tests were completed in the seated position except for the beginning of the test, when athletes could get out of the seat until on top of the gear. 


\section{Respiratory gas exchange}

Each athlete's respiratory gas exchange parameters were measured throughout the 4000-m time trial using a Cortex Metalyser 3B (Biophysik, Leipzig, Germany), which has shown to be an effective and reliable gaseous exchange instrument [23]. All parameters were calculated as the average of the two highest consecutive 30 -s measurements as a result of the tests' short duration (5-8 min) in comparison to traditional $\mathrm{VO}_{2}$ max tests $(8-12 \mathrm{~min})$. The gas analyzer was calibrated before each testing day using certified gases (15\% $\mathrm{O}_{2}, 5 \% \mathrm{CO}_{2}$ ) and the flow turbine was calibrated before each individual time trial using a $3 \mathrm{~L}$ syringe as previously described [10]. Time to completion (s), average power (W), relative and absolute $\mathrm{VO}_{2}$ peak ( $\mathrm{mL} \cdot \mathrm{min}^{-1} \cdot \mathrm{kg}^{-1}$ and $\mathrm{L} \cdot \mathrm{min}^{-1}$, respectively), and respiratory exchange ratio (RER) were all measured.

\section{Lactate, heart rate and perceived exertion}

Lactate (LactatePro, Arkray, Kyoto, Japan) was collected using a fingertip capillary blood sample pre-warm-up as well as immediately post and 5-min post each time trial to attempt to capture the peak reading. Similar collection times have been used by other researchers [24]. Heart rate was measured continuously (Polar, Kempele, Finland) during the time trial and a finishing heart rate (bpm) was measured. Immediately upon completion of the time trial each athlete provided a rating of perceived exertion (RPE) and this was reported individually to prevent bias [5] ( Table 2 ).

\section{Ischemic preconditioning application}

The protocols commenced the morning following the first 4000$\mathrm{m}$ time trial. With the subject in the supine position, a manual inflatable cuff (East Shore Medical Supply Inc., New York, USA) was positioned unilaterally on the upper leg where the adductor longus muscle attaches to the inguinal ligament. A line was drawn with a permanent marker perpendicular to the femur from this junction to ensure the inflatable cuff was positioned in exactly the same position each day. Athletes allocated to the once-a-day treatment (40 $\mathrm{min}$ ) received $4 \times 5 \mathrm{~min}$ occlusion $/ 5 \mathrm{~min}$ reperfusion of IP $(220 \mathrm{mmHg}$ ) per leg [19] in the morning followed by $4 \times 5$ min occlusion/5 min reperfusion episodes of sham treatment $(20 \mathrm{mmHg})$ per leg in the evening. The pressures chosen have been commonly used in IP research protocols $[6,19]$. Allocation to the twice-a-day group ( $80 \mathrm{~min}$ ) required the same $220 \mathrm{mmHg}$ treatment as the once-a-day group but was administered both in the morning and evening. The placebo group ( $0 \mathrm{~min}$ at $220 \mathrm{mmHg}$ ) was administered the same sham treatment $(20 \mathrm{mmHg})$ as the once-a-day group but in the morning and evening. Alternating unilateral occlusion was achieved by alternating the inflatable cuff from the left to the right leg (when one leg received reperfusion, the other was under ischemia) to ensure the volume of tissue occluded was great enough to elicit a physiological response [17]. Each athlete completed their allocated treatment at approximately the same time of day for seven consecutive days at their own residence, and correct administration was provided by the research team following the first experimental day.

\section{Statistical analyses}

The effect of repeated IP duration on the change in aerobic capacity parameters was tested in a linear mixed-effects model fitted

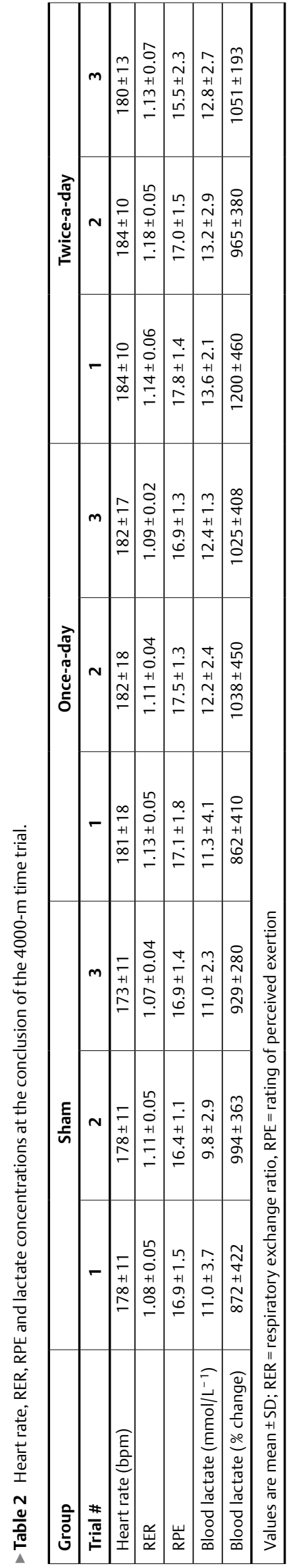


with restricted maximum likelihood, conducted in the Ime4 package [3], in R version 3.1.1. In conjunction with Cohen's effect sizes (d), P values were calculated using Satterthwaite's method of denominator synthesis, conducted in the ImerTest package [18] for R. Each aerobic capacity parameter was analyzed as the response variable in a separate model. Athlete identity was included as crossed random effects to account for the non-independence of marker measures from each athlete. All data is presented as mean \pm SEM. Statistical significance was set at $p<0.05$.

\section{Results}

Repeated IP did not result in any change in 4000-m time-trial time ( $>$ Fig. 2a) $24 \mathrm{~h}$ and seven days post-treatment for the sham $(p=0.2$ to $0.5, d=0.05$ to 0.22 ) and once-a-day ( $p=0.3$ to $0.6, d=0.06$ to 0.09 ) protocols. It did however result in a significant but trivial increase in time-trial time (performance detriment) immediately post $(p=0.03, d=0.17)$ and a non-significant trivial change (performance detriment) seven days post $(p=0.07, d=0.14)$ for the twice- a-day IP group. Individual differences did exist within each of the treatment groups for the 4000-m time-trial time. However, no differences existed between or within the three treatment groups for finishing heart rate, RER and RPE $(p>0.05, d<0.2)$ following the 4000-m time trials.

There was also no difference in the average power ( $\triangleright$ Fig. $2 \mathbf{b}$ ) for the sham ( $p=0.3$ to $0.4, d=0.07$ to 0.1 ) and once-a-day ( $p=0.4$ to $0.9, d=0.0$ to 0.15 ) IP protocols immediately and seven days post IP but a significant small decrease ( $p=0.03$ to $0.04, d=0.23$ ) for the twice-a-day IP protocol (performance detriment).

There was a trend $(p=0.07, d=0.1)$ toward an increase in relative $\mathrm{VO}_{2}$ peak ( $\triangleright$ Fig. 3 a) for the sham group immediately following the repeated IP protocol and a significant small increase seven days post $(p=0.02, d=0.24)$, which resulted in a significant small increase $(p=0.01, d=0.27)$ in absolute $\mathrm{VO}_{2}$ peak ( $>$ Fig. $\mathbf{3 b}$ ). No differences existed for relative and absolute $\mathrm{VO}_{2}$ peak for the once-a-day $(p>0.05, d<0.1)$ and twice-a-day $(p>0.05, d<0.1)$ IP protocols at any time point during the time trials.
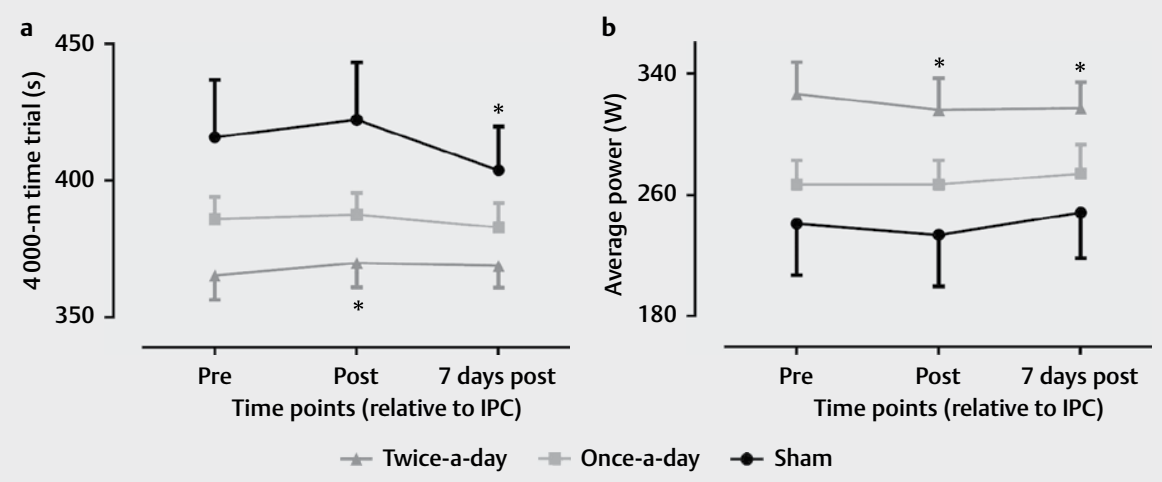

Fig. 2 4000-m time-trial time (a) and average power (b) for each of the repeated ischemic preconditioning protocols. (Sham $=0 \mathrm{~min} / \mathrm{day}$ at $220 \mathrm{mmHg}$; once-a-day $=40 \mathrm{~min} /$ day at $220 \mathrm{mmHg}$; twice-a-day $=80 \mathrm{~min} /$ day at $220 \mathrm{mmHg}$ ). Data are mean + SEM. ${ }^{*} \mathrm{p}<0.05$ for the within-group change from the pretest.
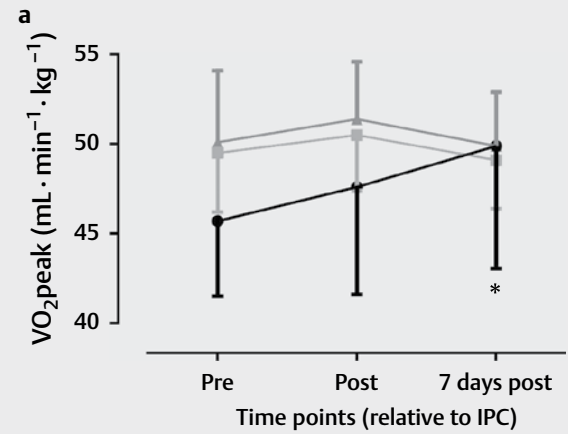

$\rightarrow$ Twice-a-day b

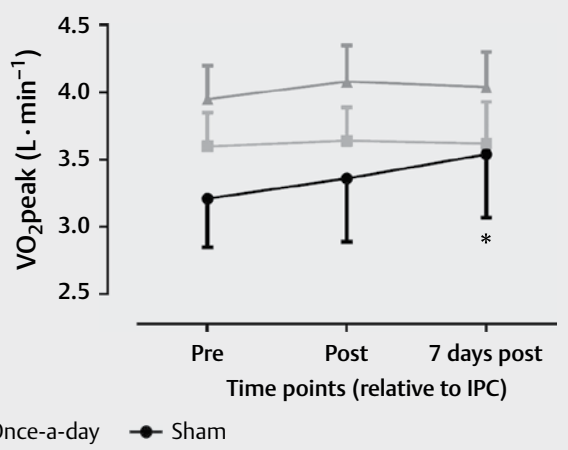

Fig. 3 Relative (a) and absolute (b) $\mathrm{VO}_{2}$ peak following the 4000-m time-trial times for the repeated ischemic preconditioning protocols. (Sham $=0 \mathrm{~min} /$ day at $220 \mathrm{mmHg}$; once-a-day $=40 \mathrm{~min} /$ day at $220 \mathrm{mmHg}$; twice-a-day $=80 \mathrm{~min} /$ day at $220 \mathrm{mmHg}$ ). Data are mean + SEM. ${ }^{*} \mathrm{p}<0.05$ for the within-group change from the pretest. 
No statistical difference existed between or within the three treatment groups for absolute and percentage changes in blood lactate concentrations $(p>0.05, d<0.1)$ following each of the 4000-m time trials.

\section{Discussion}

This study is unique because it is the first to investigate the benefit of doubling the ischemia-reperfusion tissue exposure in moderately trained athletes. However, in contrast to previous findings $[19,9]$ but in agreement with other researchers [24, 27], there was no improvement in $\mathrm{VO}_{2}$ peak or cycling performance for the groups administered IP. This raises the possibility of responder versus nonresponders to IP [14]. Indeed, if we look at individual responses within the groups of the present study, we find that the once-a-day and twice-a-day groups did have two and three responders, respectively. Hence, when the results are grouped with the non-responders in each group, overall group changes may be masked. Sex has also been suggested to influence the success rate of IP investigations, with IP less effective in female populations [11]. There were three and two females in the once- and twice-a-day groups, respectively, thereby increasing the difficulty of seeing positive changes in each of these groups.

A previous IP study undertaken [19] used recreationally active participants, many of whom were unfamiliar with cycling, and any form of training stimulus would likely result in functional adaptation. The moderately trained cyclists of the present investigation competed at a national standard in their respective age categories, and were actively training on average $10.3 \pm 3$.6 h a week. The 'ceiling effect' may have an influence here with these athletes having less scope to improve, and indeed may require a larger IP stimulus to show even a small adaptation gain as previously suggested [13]. Interestingly, other studies that used IP acutely (single session) have seen performance improvements in sports such as cycling [9], Olympic level swimming [15] and running [1], which indicates other factors may be have contributed to the lack of the present response.

Another possibility is the IP combined with the athletes current training phase resulted in too great a stress and subsequent maladaptation. The sham group showed significant albeit small gains in both relative and absolute $\mathrm{VO}_{2}$ peak in the retest 7 days post, whereas the IP intervention groups showed no change. Evidence suggests, however, that alterations in key inflammatory cytokines and modulation of oxidative mechanisms are partially responsible for the protective effect of acute and repeated IP [4]. The combined effect of these changes in combination with exercise-induced stress may lead to a physiological environment that inhibits a positive training adaptation through development of negative stimulus. Indeed, application of a repeated IP protocol has been shown to negate vascular protection in an animal model [7].

Rather than using a maximal exercise test, the current study aimed to provide more applicable event-specific advice by quantifying the potential performance benefit of using a set cycling event, which in this case equated to a 4000-m time-trial distance. Although the participants were competitive track cyclists, several limitations may have contributed to the non-significant group differences. During the time trials, minimal feedback was given to the athletes, yet in competition these athletes may rely on many sources of external feedback including sideline coaches, clocks, and even their opposition to optimize their pacing strategy. It has been previously recommended that athletes need time to adjust to the perceptual experience of endurance time trials [29]. The current finding is in agreement with a study by Tocco et al. (2015) conducted on runners that found IP did not improve self-paced exercise performance [32].

Our study demonstrated that repeated IP does not improve 4000-m time-trial performance, irrespective of the dose and the number of days after the intervention the cyclist is tested. Previously in our lab, we observed that repeated IP improves lactate clearance; however on this occasion there was no change. It is possible that a positive lactate clearance effect was attenuated in these conditioned athletes when training is combined with the repeated IP dose. Coaches may suggest that IP could provide a useful training stimulus even for those highly conditioned in certain situations or during certain phases of training, such as returning from injury or in the peaking phase before a major competition when the training volume is dramatically reduced. However, studies are required to confirm any potential gains of IP used repeatedly as a training tool. Until then, the validity of using IP as a training tool to enhance athletic performance is questionable. Any future studies should look to determine the optimal dosage, especially for individuals, by manipulating three main variables: the number of occlusion bouts per session, the duration of each occlusion repetition and the number of days over which the protocol is performed. Finally, new markers of adaptation should also be investigated to allow monitoring of IP progress within any intervention phase.

Although IP used as a training tool through repeated daily administration has previously been shown successful in inducing functional changes that have the potential to improve sporting performance, further research is required to optimize the required dosage. Individualization and timing of the dose may be required to maximize adaptation and subsequent sporting performance.

\section{Acknowledgements}

The authors wish to thank Rebecca Freebairn, Robin Vis and Morgan Fitzgerald for their dedicated help during data collection and each of the subjects for their participation.

\section{Conflict of Interest}

The authors declare that they have no conflict of interest.

\section{References}

[1] Bailey TG, Jones H, Gregson W, Atkinson G, Cable NT, Thijssen DHJ. Effect of ischemic preconditioning on lactate accumulation and running performance. Med Sci Sports Exerc 2012; 44: 2084-2089

[2] Banks L, Wells GD, Clarizia NA, Jean-St-Michel E, McKillop AL, Redington AN, McCrindle BW. Short-term remote ischemic preconditioning is not associated with improved blood pressure and exercise capacity in young adults. Appl Physiol Nutr Metab 2016; 41: 903-906 
[3] Bates D, Maechler M, Bolker B, Walker MS.Package 'mlmRev'. Examples from multilevel modelling software review 2014; https:// cran.r-project.org/web/packages/mlmRev/mlmRev.pdf

[4] Berger MM, Macholz F, Mairbäurl H, Bärtsch P. Remote ischemic preconditioning for prevention of high-altitude diseases: Fact or fiction? J Appl Physiol 2015; 119: 1143-1151

[5] Borg GA. Psychophysical bases of perceived exertion. Med Sci Sports Exerc 1982; 14: 377-381

[6] Cocking S, Wilson MG, Nichols D, Cable NT, Green D], Thijssen DH], Jones $\mathrm{H}$. Is there an optimal ischemic-preconditioning dose to improve cycling performance? Int J Sports Physiol Perform 2018; 13: 274-282

[7] Cohen MV, Yang X-M, Downey JM. Conscious rabbits become tolerant to multiple episodes of ischemic preconditioning. Circ Res 1994; 74 : 998-1004

[8] Crisafulli A, Tangianu F, Tocco F, Concu A, Mameli O, Mulliri G, Caria MA. Ischemic preconditioning of the muscle improves maximal exercise performance but not maximal oxygen uptake in humans. J Appl Physiol 2011; 111: 530-536

[9] De Groot PCE, Thijssen DH], Sanchez M, Ellenkamp R, Hopman MTE. Ischemic preconditioning improves maximal performance in humans. Eur J Appl Physiol 2010; 108: 141

[10] Foster GP, Giri PC, Rogers DM, Larson SR, Anholm JD. Ischemic preconditioning improves oxygen saturation and attenuates hypoxic pulmonary vasoconstriction at high altitude. High Alt Med Biol 2014; 15: 155-161

[11] Gibson N, White J, Neish M, Murray A. Effect of ischemic preconditioning on land-based sprinting in team-sport athletes. Int J Sports Physiol Perform 2013; 8: 671-676

[12] Harriss D], Macsween A, Atkinson G. Standards for ethics in sport and exercise science research: 2018 update. Int J Sports Med 2017; 38: $1126-1131$

[13] Hittinger EA, Maher JL, Nash MS, Perry AC, Signorile JF, Kressler ], Jacobs KA. Ischemic preconditioning does not improve peak exercise capacity at sea level or simulated high altitude in trained male cyclists. Appl Physiol Nutr Metab 2014; 40: 65-71

[14] Incognito AV, Burr JF, Millar PJ. The effects of ischemic preconditioning on human exercise performance. Sports Med 2016; 46: 531-544

[15] Jean-St-Michel E, Manlhiot C, Li J, Tropak M, Michelsen MM, Schmidt MR, McCrindle BW, Wells GD, Redington AN. Remote preconditioning improves maximal performance in highly trained athletes. Med Sci Sports Exerc 2011; 43: 1280-1286

[16] Jones H, Hopkins N, Bailey TG, Green DJ, Cable NT, Thijssen DHJ. Seven-day remote ischemic preconditioning improves local and systemic endothelial function and microcirculation in healthy humans. Am J Hypertens 2014; 27: 918-925

[17] Kraus AS, Pasha EP, Machin DR, Alkatan M, Kloner RA, Tanaka H. Bilateral upper limb remote ischemic preconditioning improves anaerobic power. Open Sports Med J 2015; 9: 1-6

[18] Kuznetsova A, Brockhoff PB, Christensen RHB. ImerTest: Tests for random and fixed effects for linear mixed effect models (Imer objects of Ime4 package). R package version 2: 0-0 2013
[19] Lindsay A, Petersen C, Blackwell G, Ferguson H, Parker G, Steyn N, Gieseg SP. The effect of 1 week of repeated ischaemic leg preconditioning on simulated Keirin cycling performance: A randomised trial. BMJ Open Sport Exerc Med 2017; 3: e000229

[20] Marocolo M, da Mota GR, Pelegrini V, Appell Coriolano H]. Are the beneficial effects of ischemic preconditioning on performance partly a placebo effect? Int J Sports Med 2015; 36: 822-825

[21] Marocolo M, Marocolo IC, da Mota GR, Simao R, Maior AS, Coriolano $\mathrm{HJ}$. Beneficial effects of ischemic preconditioning in resistance exercise fade over time. Int J Sports Med 2016; 37: 819-824

[22] Marocolo M, Willardson JM, Marocolo IC, da Mota GR, Simão R, Maior AS. Ischemic preconditioning and placebo intervention improves resistance exercise performance. Journal Strength Cond Res 2016; 30 : 1462-1469

[23] Meyer T, Davison RCR, Kindermann W. Ambulatory gas exchange measurements-current status and future options. Int I Sports Med 2005; 26: S19-S27

[24] Paixao RC, da Mota GR, Marocolo M. Acute effect of ischemic preconditioning is detrimental to anaerobic performance in cyclists. Int J Sports Med 2014; 35: 912-915

[25] Paradis-Deschenes $P$, Joanisse DR, Billaut F. Ischemic preconditioning improves time trial performance at moderate altitude. Med Sci Sports Exerc 2018; 50: 533-541

[26] Patterson SD, Bezodis NE, Glaister M, Pattison JR. The effect of ischemic preconditioning on repeated sprint cycling performance. Med Sci Sports Exerc 2015; 47: 1652-1658

[27] Sabino-Carvalho JL, Lopes TR, Obeid-Freitas T, Ferreira TN, Succi JE, Silva AC, Silva BM. Effect of ischemic preconditioning on endurance performance does not surpass placebo. Med Sci Sports Exerc 2017; 49: 124-132

[28] Salvador AF, De Aguiar RA, Lisbôa FD, Pereira KL, Cruz RSdO, Caputo F. Ischemic preconditioning and exercise performance: A systematic review and meta-analysis. Int J Sports Physiol Perform 2016; 11: 4-14

[29] Schmit C, Duffield R, Hausswirth C, Coutts A], Le Meur Y. Pacing adjustments associated with familiarization: Heat versus temperate environments. Int J Sports Physiol Perform 2016; 11 : 855-860

[30] Sporer BC, McKenzie DC. Reproducibility of a laboratory-based 20-km time trial evaluation in competitive cyclists using the Velotron Pro ergometer. Int J Sports Med 2007; 28: 940-944

[31] Stone MR, Thomas K, Wilkinson M, Gibson ASC, Thompson KG. Consistency of perceptual and metabolic responses to a laboratorybased simulated 4,000-m cycling time trial. Eur J Appl Physiol 2011; 111: 1807-1813

[32] Tocco F, Marongiu E, Ghiani G, Sanna I, Palazzolo G, Olla S, Pusceddu M, Sanna P, Corona F, Concu A. Muscle ischemic preconditioning does not improve performance during self-paced exercise. Int J Sports Med 2015; 36: 9-15 